\title{
Chains and antichains in Boolean algebras
}

\author{
by
}

M. L o s a d a and S. Tod or čevi ć (Toronto and Paris)

\begin{abstract}
We give an affirmative answer to problem DJ from Fremlin's list ([8]) which asks whether $\mathrm{MA}_{\omega_{1}}$ implies that every uncountable Boolean algebra has an uncountable set of pairwise incomparable elements.
\end{abstract}

1. Introduction. Every Boolean algebra $\mathcal{B}$ has a natural ordering which is especially apparent when we think of $\mathcal{B}$ as a field of subsets of some set. In this case the ordering of $\mathcal{B}$ is simply the inclusion relation $\subseteq$ and one usually keeps this notation even in the situation when the representation of $\mathcal{B}$ as a field of sets is not explicit. Two elements $a$ and $b$ in $\mathcal{B}$ are comparable if either $a \subseteq b$ or $b \subseteq a$; otherwise we say that $a$ and $b$ are incomparable. The comparability relation on $\mathcal{B}$ can sometimes be used when finding distinctions between various classes of Boolean algebras. For example, if $\mathcal{B}$ is a free algebra then, regardless of its size, the order relation is not very present in $\mathcal{B}$. An old result of Galvin and Jónsson (see [9]) states that no free lattice $\mathcal{B}$ can have an uncountable chain and, in fact, that there is a decomposition $\mathcal{B}=\bigcup_{n=0}^{\infty} \mathcal{B}_{n}$ such that for every $n$, any two distinct elements of $\mathcal{B}_{n}$ are incomparable (we will generally call a set of pairwise incomparable elements of $\mathcal{B}$ an antichain of $\mathcal{B})$. On the other hand, Bonnet and Shelah [5] and the second author [16] have constructed examples of chains $L \subseteq \mathcal{P}(\omega)$ such that the Boolean subalgebra $\mathcal{B}(L)$ of $\mathcal{P}(\omega)$ generated by $L$ does not have an antichain of size equal to the size of $L$. So in this case the comparability relation is much more present than incomparability. However, an examination of the class of interval algebras (such as $\mathcal{B}(L)$ above) shows that no uncountable interval algebra can be represented as the union of countably many chains. Thus, while one cannot expect that every uncountable Boolean algebra has an uncountable chain, it is still possible to ask whether every uncountable Boolean algebra contains an uncountable antichain. The afore-

2000 Mathematics Subject Classification: 03E50, 06E10, 06E15.

Research of the second author is partially supported by SFS and NSERC. 
mentioned examples of Bonnet-Shelah [5] and the second author [16] show that an affirmative answer to this question must involve an extra axiom of set theory since the Continuum Hypothesis $(\mathrm{CH})$ gives a counterexample. An educated guess is that a natural candidate for such an axiom is Martin's axiom, $\mathrm{MA}_{\omega_{1}}$ (see [7]). A major breakthrough in this area was made by Baumgartner [2] when he constructed a model of $\mathrm{MA}_{\omega_{1}}$ in which every uncountable Boolean algebra contains an uncountable antichain. After the discovery of the Proper Forcing Axiom, PFA, he pointed out that his proof in fact shows that PFA is an extra axiom of set theory that implies that every uncountable Boolean algebra contains an uncountable antichain (see [3]). However, this still left open whether $\mathrm{MA}_{\omega_{1}}$ itself is strong enough to give the same conclusion.

The related problem, asking whether every uncountable partially ordered set must contain either an uncountable chain or an uncountable antichain, is much older. A well-known example of Sierpiński and Kurepa ([14], [10]) is an actual counterexample. It is an ordering of $\mathbb{R}$ obtained by intersecting the usual ordering with a well-ordering. An unpublished result of Galvin, showing that no uncountable subset of the Sierpinski poset can be represented as $(X, \subseteq)$ for some $X \subseteq \mathcal{P}(\omega)$, hinted that the question of chains versus antichains should really be restricted to the class of suborderings of $\mathcal{P}(\omega)$. This indeed turned out to be the right approach since Baumgartner's proof (see [3]) also shows that PFA implies that every uncountable subset of $\mathcal{P}(\omega)$ contains an uncountable chain or an uncountable antichain; a statement which almost immediately (see [2] or [3]) implies that every uncountable Boolean algebra contains an uncountable antichain. Thus a question that naturally arises again is whether $\mathrm{MA}_{\omega_{1}}$ itself is sufficient to give us the same conclusion.

Interestingly enough, $\mathrm{MA}_{\omega_{1}}$ does not imply that every uncountable subset $\mathcal{X}$ of $\mathcal{P}(\omega)$ must contain an uncountable chain or an uncountable antichain. This follows from a result of Abraham and Shelah [1] which states that $\mathrm{MA}_{\omega_{1}}$ is consistent with the existence of a one-to-one function $f$ from some uncountable set $A \subseteq \mathbb{R}$ into $\mathbb{R}$ which is not monotonic on any uncountable subset of $A$. To see this, note that $(\mathbb{R}, \subseteq)$ is isomorphic to a chain in $(\mathcal{P}(\omega), \subseteq)$ via the embedding

$$
r \mapsto\left\{n: q_{n}<r\right\}
$$

for some fixed enumeration $\left\{q_{n}: n<\omega\right\}$ of the rationals. Thus we can find two uncountable chains $A_{0} \subseteq \mathcal{P}(2 \mathbb{N}+1)$ and $B_{0} \subseteq \mathcal{P}(2 \mathbb{N})$ and a one-to-one map $f: A_{0} \rightarrow B_{0}$ which is not monotonic on any uncountable subset of $A_{0}$. Then

$$
\mathcal{X}_{f}=\left\{a \cup(2 \mathbb{N} \backslash f(a)): a \in A_{0}\right\}
$$

is a subposet of $(\mathcal{P}(\mathbb{N}), \subseteq)$ without uncountable chains or uncountable an- 
tichains. Note that such an $f$, and therefore such an $\mathcal{X}_{f}$, can be constructed using $\mathrm{CH}$. Even more, one can use $\mathrm{CH}$ to get a $\mathcal{P}(\omega)$-analogue of the Sierpiński poset, that is, an uncountable well-founded subset of $\mathcal{P}(\omega)$ without uncountable antichains (or chains). This was first done by Baumgartner and Kunen, see [2]. In fact, there is a ZFC-example of an uncountable well-founded subset $\mathcal{X}$ of $\mathcal{P}(\omega)$ which contains no antichain of size equal to the size of $\mathcal{X}$ (see [17]; Lemma 16). On the other hand, Boolean-algebraic analogues of the Sierpiński poset have also been constructed by Baumgartner and Komjáth [4] using $\diamond$, and by Shelah [13] using $\mathrm{CH}$, exhibiting uncountable Boolean algebras without uncountable chains or uncountable antichains.

The purpose of this paper is to show that $\mathrm{MA}_{\omega_{1}}$ is indeed a sufficiently strong additional axiom of set theory to imply that every uncountable Boolean algebra contains an uncountable antichain, showing thus a striking discrepancy between the class of uncountable Boolean algebras and the wider class of uncountable partial orderings.

2. Preliminaries. As we mentioned above, throughout this paper we address the following question.

Question 2.1. $\left(\mathrm{MA}_{\omega_{1}}\right)$ Does every uncountable Boolean algebra $\mathcal{B}$ have an uncountable set of pairwise incomparable elements?

In this section we collect some known results related to Question 2.1. We have already indicated above that the corresponding question for uncountable partially ordered sets is much older and that we can hope for a (consistent) affirmative answer only in the case of uncountable suborderings of $(\mathcal{P}(\omega), \subseteq)$. We shall need a general fact about the effect of $\mathrm{MA}_{\omega_{1}}$ on such suborderings of $\mathcal{P}(\omega)$. To state it we need the following definition.

Definition 2.2. An uncountable linearly ordered set $(L, \leq)$ is called Countryman if its Cartesian square is the union of countably many chains.

Remark 2.3. It is easily seen that no set $X$ of reals is Countryman. To see this, choose $f: X \rightarrow X$ such that $f^{-1}(x)$ is uncountable for every $x \in X$. If $X^{2}$ can be covered by countably many chains then $f$ would contain a nondecreasing restriction $g=f \mid Y$ such that

$$
Z=\left\{z \in X: g^{-1}(z) \text { is uncountable }\right\}
$$

is uncountable. Note that for $y \neq z$ in $Z, g^{-1}(y)$ and $g^{-1}(z)$ are two disjoint convex subsets of $Y$. So we would be able to obtain from $g^{-1}(z)(z \in Z)$ an uncountable family of disjoint open intervals of $Y$, a contradiction.

Remark 2.4. Note also that if $(L, \leq)$ is Countryman then every finite power of $(L, \leq)$ with the coordinatewise (Cartesian) ordering is also the 
union of countably many chains. To see this, let $c: L^{2} \rightarrow \omega$ be a fixed chain-decomposition of $L^{2}$. For an integer $n \geq 2$ define $c_{n}: L^{n} \rightarrow \omega^{n^{2}}$ by letting

$$
c_{n}\left(x_{0}, \ldots, x_{n-1}\right)=\left\langle c\left(x_{i}, x_{j}\right): i, j<n\right\rangle .
$$

It is easily checked that $c_{n}$ is a chain-decomposition of $L^{n}$.

The following lemma is due to Kunen in the case of well-orderings (see [7]) and to Baumgartner [2] in the more general case of Aronszajn orderings. For our purposes we will only need the special case of Countryman orderings rather than Aronszajn and, in order to make this paper self-contained, we give a proof of this special case.

Lemma 2.5. $\left(\mathrm{MA}_{\omega_{1}}\right)$ Suppose $X \subseteq \mathcal{P}(\omega)$ is uncountable and there exists a strictly increasing map $f:(X, \subseteq) \rightarrow(L, \leq)$, where $L$ is either a well-order or a Countryman order. Then $X$ has an uncountable antichain.

Proof. Our assumption on $X \subseteq \mathcal{P}(\omega)$ implies that there is a total ordering $\preceq$ on $X$ such that $(X, \preceq)$ is either a well-ordered set or a Countryman ordering and such that $x \subseteq y$ implies $x \preceq y$ for all $x, y \in X$. In fact, the only property of $(X, \preceq)$ that we need to complete the proof (besides the fact that it does not contain an uncountable subordering isomorphic to the reals) is the property that every uncountable subset of some finite power of $(X, \preceq)$ contains a chain under the coordinatewise ordering. Let $\mathcal{P}$ be the poset of all finite antichains of $X$ ordered by inclusion. By $\mathrm{MA}_{\omega_{1}}$ it suffices to show that $\mathcal{P}$ is a c.c.c. forcing notion. Otherwise, using a standard $\Delta$-system argument one can find an integer $n$ and an uncountable family $\mathcal{F}$ of pairwise disjoint subsets of $X$, all of size $n$, such that $F \cup G$ is not an antichain of $(X, \subseteq)$ for all $F \neq G$ in $\mathcal{F}$. Note that since an element $F$ of $\mathcal{F}$ is an antichain of $(X, \subseteq)$ there is a $k=k_{F}<\omega$ such that $\{x \cap\{0, \ldots, k-1\}: x \in F\}$ is also an antichain of size $n$. Going to an uncountable subfamily of $\mathcal{F}$ we may assume that there is a single integer $k$ and a single antichain $\left\{t_{0}, \ldots, t_{n-1}\right\}$ of $n$ subsets of $\{0, \ldots, k-1\}$ such that for every $F \in \mathcal{F}$ if $\left\{x_{0}^{F}, \ldots, x_{n-1}^{F}\right\}$ is an $\preceq$-increasing enumeration of $F$ then

$$
x_{i}^{F} \cap\{0, \ldots, k-1\}=t_{i} \quad \text { for } i<n .
$$

It follows that for $F \neq G$ in $\mathcal{F}$ there is an $i<n$ such that either $x_{i}^{F} \subseteq x_{i}^{G}$ or vice versa, $x_{i}^{G} \subseteq x_{i}^{F}$. Using the assumption on the total ordering $\preceq$ we may assume, by going to an uncountable subset of $\mathcal{F}$ and identifying each $F$ in $\mathcal{F}$ with $\left\langle x_{0}^{F}, \ldots, x_{n-1}^{F}\right\rangle$, that $\mathcal{F}$ is a chain in the Cartesian $n$-power of $(X, \preceq)$. For $F \in \mathcal{F}$, set

$$
C_{F}=\left\{\left\langle x_{0}, \ldots, x_{n-1}\right\rangle \in \mathcal{P}(\omega)^{n}: x_{i} \subseteq x_{i}^{F} \text { for some } i<n\right\} .
$$

Considering $\mathcal{P}(\omega)^{n}$ as the topological $n$-power of the Cantor space we see that each $C_{F}$ is a closed subset of the power. Note that if $F \neq G$ are 
members of the family $\mathcal{F}$ then

$$
x_{0}^{F} \prec x_{0}^{G} \quad \text { implies }\left\langle x_{0}^{F}, \ldots, x_{n-1}^{F}\right\rangle \in C_{G} \quad \text { but }\left\langle x_{0}^{G}, \ldots, x_{n-1}^{G}\right\rangle \notin C_{F} \text {. }
$$

It follows that $\left(X_{0}=\left\{x_{0}^{F}: F \in \mathcal{F}\right\}, \preceq\right)$ is isomorphic to a chain

$$
\left(\left\{C_{F} \cap \mathcal{F}: F \in \mathcal{F}\right\}, \subseteq\right)
$$

of closed subsets of $\mathcal{F}$, when $\mathcal{F}$ is considered as a separable metric space with the topology induced from $\mathcal{P}(\omega)^{n}$. Hence, in particular, $\left(X_{0}, \preceq\right)$ is isomorphic to a set of reals with the usual ordering, a contradiction. The lemma follows.

In general, it will be useful to restrict ourselves to $\mathcal{P}(\omega)$ when answering Question 2.1; the precise way is stated in the following well-known lemma ([4] or [11]).

LEMMA 2.6. Every Boolean algebra $\mathcal{B}$ without an uncountable set of pairwise incomparable elements is isomorphic to a subalgebra of the power set $\mathcal{P}(\omega)$.

Proof. Note that the conclusion of the lemma is equivalent to the statement that $\mathcal{B}$ is $\sigma$-centered, or equivalently that the Stone space of $\mathcal{B}$ is separable. Otherwise, since $\mathcal{B}$ is a c.c.c. algebra, we can find disjoint $a, b \in \mathcal{B}^{+}=\mathcal{B} \backslash\{0\}$ such that neither of the restrictions $\mathcal{B}^{+}\left\lceil a\right.$ and $\mathcal{B}^{+} \uparrow b$ is $\sigma$-centered. Using this recursively on countable ordinals, pick sequences

$$
\left\{a_{\alpha}: \alpha<\omega_{1}\right\} \subseteq \mathcal{B}^{+}\left\lceil a \quad \text { and } \quad\left\{b_{\alpha}: \alpha<\omega_{1}\right\} \subseteq \mathcal{B}^{+} \uparrow b\right.
$$

such that $a_{\beta} \subseteq a_{\alpha}$ or $b_{\beta} \subseteq b_{\alpha}$ can only happen when $\beta>\alpha$. Let

$$
\mathcal{X}=\left\{a_{\alpha} \cup\left(b \backslash b_{\alpha}\right): \alpha<\omega_{1}\right\} .
$$

Clearly, $\mathcal{X}$ is an uncountable antichain of $\mathcal{B}$. The lemma follows.

REMARK 2.7. Note that the argument shows that in Lemma 2.6 we have a stronger conclusion: $\mathcal{B}$ has a countable order-dense subset.

Therefore, without loss of generality, we may assume that $\mathcal{B}$ is a subalgebra of $\mathcal{P}(\omega)$. Furthermore we may assume that $\mathcal{B}$ does not have an uncountable subset satisfying the conditions of Lemma 2.5. In particular, this will mean that all ideals of subalgebras of $\mathcal{B}$ are countably generated. For assume that the ideal $\mathcal{I} \subseteq \mathcal{B}$ is not countably generated. Then we can construct inductively a sequence $S=\left\langle x_{\gamma}: \gamma<\omega_{1}\right\rangle \subseteq \mathcal{I}$ such that for each $\alpha \neq \beta<\omega_{1}$, if $x_{\alpha} \subseteq x_{\beta}$ then $\alpha<\beta$. When this happens we say that the inclusion relation on $S$ is extended by the well-ordering on its index set $\omega_{1}$. In other words, there is a strictly increasing map from $S$ into $\omega_{1}$ which, by Lemma 2.5 , implies that $\mathcal{B}$ has an uncountable family of pairwise incomparable sets. 
The following lemma is also a part of the folklore and gives another restriction on $\mathcal{B}$ that we may assume when trying to answer Question 2.1.

Lemma 2.8. $\left(\mathrm{MA}_{\omega_{1}}\right)$ Let $\mathcal{B}$ be an uncountable Boolean algebra with an uncountable chain. Then $\mathcal{B}$ has an uncountable antichain.

Proof. Let $L$ be a fixed uncountable chain in $\mathcal{B}$. Recall that we may assume that $\mathcal{B}$ is a subalgebra of $\mathcal{P}(\omega)$. It follows that $L$ has a countable order-dense subset $D$. Note that there must be an element $d$ in $D$ such that $L_{0}=\{x \in L: x \subset d\}$ and $L_{1}=\{x \in L: d \subset x\}$ are both uncountable. Pick an arbitrary one-to-one mapping $f: L_{0} \rightarrow L_{1}$. (If $L_{1}$ has smaller size than $L_{0}$ we switch the roles of $L_{0}$ and $L_{1}$.) If there is an uncountable set $K \subseteq L_{0}$ such that $f\lceil K$ is increasing then $\{x \cup(-f(x)): x \in K\}$ would be an uncountable antichain of $\mathcal{B}$. We can obtain such a $K$ by applying $\mathrm{MA}_{\omega_{1}}$ to the poset $\mathcal{P}$ of all finite subsets $p$ of $L_{0}$ such that $f\lceil p$ is increasing. For this we need to assume that $\mathcal{P}$ satisfies the countable chain condition (c.c.c.). So the lemma would be proved once we show the following:

Claim 2.9. If $\mathcal{P}$ is not c.c.c. then $\mathcal{B}$ contains an uncountable set of pairwise incomparable elements.

P r o of. Assume we can choose an uncountable sequence $\left\langle p_{\alpha}: \alpha<\omega_{1}\right\rangle$ of pairwise incompatible elements of $\mathcal{P}$. Using a standard $\Delta$-system argument we may assume, by refining and removing the root, that the $p_{\alpha}$ 's are disjoint and have all the same size $n$. For each $\alpha$ we fix sequences $I_{\alpha}^{0}, \ldots, I_{\alpha}^{n-1}$ and $J_{\alpha}^{0}, \ldots, J_{\alpha}^{n-1}$ of disjoint closed intervals with endpoints in $D$ which separate $p_{\alpha}$ and its image $f^{\prime \prime} p_{\alpha}$, respectively. In other words, for each $i<n$ there is a unique element $x_{\alpha}^{\prime}$ in $p_{\alpha} \cap I_{\alpha}^{i}$ whose image $f\left(x_{\alpha}^{\prime}\right)$ is the unique member of $\left(f^{\prime \prime} p_{\alpha}\right) \cap J_{\alpha}^{i}$. Since there exist only countably many such sequences of intervals, there is an uncountable set $\Omega \subseteq \omega_{1}$ and $I^{0}, \ldots, I^{n-1}$ and $J^{0}, \ldots, J^{n-1}$ such that $I_{\alpha}^{i}=I^{i}$ and $J_{\alpha}^{i}=J^{i}$ for all $\alpha \in \Omega$ and $i<n$. Let $d_{i}=\min I^{i}$ and $e_{i}=\min J^{i}(i<n)$. Finally, for $\alpha \in \Omega$, let

$$
\begin{aligned}
a_{\alpha}= & \left(x_{\alpha}^{0} \backslash d_{0}\right) \cup \ldots \cup\left(x_{\alpha}^{n-1} \backslash d_{n-1}\right) \\
& \cup\left(f\left(x_{\alpha}^{0}\right) \backslash e_{0}\right) \cup \ldots \cup\left(f\left(x_{\alpha}^{n-1}\right) \backslash e_{n-1}\right) .
\end{aligned}
$$

For $\alpha \neq \beta$ in $\Omega$ the fact that $p_{\alpha}$ and $p_{\beta}$ are incompatible in $\mathcal{P}$ means that $f$ is not increasing on the union $p_{\alpha} \cup p_{\beta}$, which in turn amounts to the fact that $a_{\alpha} \nsubseteq a_{\beta}$ and $a_{\beta} \nsubseteq a_{\alpha}$. This proves Claim 2.9, and hence also Lemma 2.8 .

The rest of the paper contains an approach to Question 2.1 which is briefly described as follows. In Section 3 we begin the study of a general $\mathcal{B}$ and the natural poset $\mathcal{P}$ of all finite antichains of $\mathcal{B}$. Since we are assuming $\mathrm{MA}_{\omega_{1}}$, the c.c.c.-ness of this poset $\mathcal{P}$ will give us an affirmative answer to Question 2.1. Hence, assuming the poset $\mathcal{P}$ is not c.c.c. we try to find an 
uncountable antichain of $\mathcal{B}$ using some Boolean combinations of elements of some fixed uncountable set of pairwise incompatible elements of $\mathcal{P}$, in a way similar to the proof of Lemma 2.8. Sections 4 and 5 both pertain to the case where a specific setting like this (arrived at in Section 3) cannot be attained. In this case it will be useful to work with the Stone space of our Boolean algebra $\mathcal{B}$. Auxiliary orders will be defined on a partition of the Stone space of $\mathcal{B}$ that will either help us define uncountable antichains or reduce our problem to Lemma 2.5 .

3. Separation. In this section we prove the following lemma which isolates a crucial object that will be considered in the rest of the paper.

LEMMA 3.1. $\left(\mathrm{MA}_{\omega_{1}}\right)$ Suppose $\mathcal{B}$ is an uncountable subalgebra of $\mathcal{P}(\omega)$ which contains no uncountable antichain. Then there is a sequence $\mathcal{X}_{0}=$ $\left\langle x_{\alpha}: \alpha<\omega_{1}\right\rangle$ of elements of $\mathcal{B}$ such that whenever $M$ is a countable elementary submodel of $H_{\mathfrak{c}^{+}}$containing $\mathcal{B}$ and $\mathcal{X}_{0}$, and $b_{0}, b_{1} \in \mathcal{B} \cap M$ are disjoint, then for every $\alpha<\omega_{1}$, at least one of $x_{\alpha} \cap b_{0}$ or $x_{\alpha} \cap b_{1}$ belongs to $\mathcal{B} \cap M$.

Proof. It is natural to consider the following partial order, where $\mathcal{X}$ is an arbitrary uncountable subset of $\mathcal{B}$.

Definition 3.2. Let $\mathcal{P}=\mathcal{P}(\mathcal{X})$ be the partial order of all finite sets of pairwise incomparable elements of $\mathcal{X}$ ordered by inclusion. That is, each condition $p \in \mathcal{P}$ is of the form $p=\left\{x_{0}, \ldots, x_{m}\right\} \subseteq \mathcal{X}$ for some $m<\omega$ such that $x_{i} \nsubseteq x_{j}$ and $x_{j} \nsubseteq x_{i}$ for all $i \neq j \leq m$. If $p, q \in \mathcal{P}$ then $p$ extends $q$ if and only if $q \subseteq p$.

Note 3.3. $\mathcal{P}$ cannot be a c.c.c. poset or else we would be able to use $\mathrm{MA}_{\omega_{1}}$ on $\mathcal{P}$ and produce an uncountable antichain of $\mathcal{B}$. So let $\mathcal{A}=\left\langle p_{\alpha}=\right.$ $\left.\left\{x_{0}^{\alpha}, \ldots, x_{m_{\alpha}}^{\alpha}\right\}: \alpha<\omega_{1}\right\rangle$ be an uncountable strong antichain of $\mathcal{P}$. Using a $\Delta$-system argument to refine $\mathcal{A}$ we may assume that the $p_{\alpha}$ 's are disjoint and there exists an integer $n<\omega$ such that $m_{\alpha}=n$ for all $\alpha<\omega_{1}$.

Refining further we may assume that for a fixed $k<\omega$ and all $\alpha<\omega_{1}$, $x_{i}^{\alpha} \cap\{0, \ldots, k\} \nsubseteq x_{j}^{\alpha} \cap\{0, \ldots, k\}$ for all $i \neq j \leq n$. In fact, we may assume that for all $\alpha \neq \beta<\omega_{1}, x_{i}^{\alpha} \cap\{0, \ldots, k\}=x_{i}^{\beta} \cap\{0, \ldots, k\}$ for all $i \leq n$.

Thus for $\alpha \neq \beta<\omega_{1}$ we see that $p_{\alpha}$ is incompatible with $p_{\beta}$ if and only if there exists an $i=i_{\alpha \beta} \leq n$ such that either

$$
x_{i}^{\alpha} \subseteq x_{i}^{\beta} \quad \text { or } \quad x_{i}^{\beta} \subseteq x_{i}^{\alpha} .
$$

Since the above means that the incompatibility of two conditions of $\mathcal{A}$ can be viewed as a comparability question of their corresponding elements, it seems useful to separate the individual elements of each condition of the strong antichain according to the following definition. 
Definition 3.4. Suppose there is a sequence $\left\langle C_{0}, \ldots, C_{n}\right\rangle$ of pairwise disjoint elements of $\mathcal{B}$ and an uncountable set $\Omega \subseteq \omega_{1}$ such that

(a) $C_{i} \cap x_{i}^{\alpha} \neq \emptyset$ and $C_{i} \backslash x_{i}^{\alpha} \neq \emptyset$ for all $\alpha \in \Omega$ and $i \leq n$, and

(b) $C_{i} \cap x_{i}^{\alpha} \neq C_{i} \cap x_{i}^{\beta}$ for all $\alpha \neq \beta$ in $\Omega$ and $i \leq n$.

Then we say that the sequence $\left\langle C_{0}, \ldots, C_{n}\right\rangle$ separates $\mathcal{A}$.

A natural way to try to prove the existence of a sequence $\left\langle C_{0}, \ldots, C_{n}\right\rangle$ that would separate an uncountable subsequence of $\mathcal{A}$ is by induction on $n$. Clearly, there is no problem at stage $n=0$. So suppose we have found uncountable $\Omega_{n-1} \subseteq \omega_{1}$ and a disjoint sequence separating $\left\{\left\{x_{0}^{\alpha}, \ldots, x_{n-1}^{\alpha}\right\}\right.$ : $\left.\alpha \in \Omega_{n-1}\right\}$. If the family

$$
\left\{x_{n}^{\alpha} \backslash \bigcup_{i<n} C_{i}: \alpha \in \Omega_{n-1}\right\}
$$

of sets is uncountable we could let $C_{n}$ be the complement of $\bigcup_{i<n} C_{i}$ and refine $\Omega_{n-1}$ to $\Omega_{n}$ accordingly. Otherwise there is an $i_{0}<n$ such that

$$
\left\{x_{n}^{\alpha} \cap C_{i_{0}}: \alpha \in \Omega\right\} \text { is uncountable. }
$$

Thus we would be done if we could refine $C_{i_{0}}$ into two disjoint sets $\bar{C}_{i_{0}}$ and $\bar{C}_{n}$ that would separate $\left\{\left\{x_{i_{0}}^{\alpha}, x_{n}^{\alpha}\right\}: \alpha \in \Omega^{\prime}\right\}$ for some uncountable $\Omega^{\prime}$. This means that the problem reduces to separating strong antichains $\mathcal{A}$ of two-element conditions. Suppose this is impossible for a particular such $\mathcal{A}=\left\{\left\{x_{0}^{\alpha}, x_{1}^{\alpha}\right\}: \alpha \in \omega_{1}\right\}$.

Pick a countable elementary submodel $M$ of some large enough structure such as $H_{\mathfrak{c}^{+}}$(the collection of all sets whose transitive closures have size $\leq \mathfrak{c}$ ) such that $M$ contains $\mathcal{B}$ and $\mathcal{X}_{0}=\left\{x_{0}^{\alpha}: \alpha \in \omega_{1}\right\}$. Suppose $\delta<\omega_{1}$ is such that $\delta=M \cap \omega_{1}$. Then, in particular, for $\alpha \geq \delta$ the element $x_{0}^{\alpha}$ is not in $M$. Pick two disjoint nonempty elements $b_{0}, b_{1}$ in $\mathcal{B} \cap M$ and some $\alpha \geq \delta$. Then

$$
x_{0}^{\alpha} \cap b_{0} \in \mathcal{B} \cap M \quad \text { or } \quad x_{0}^{\alpha} \cap b_{1} \in \mathcal{B} \cap M .
$$

Otherwise, $M$ would satisfy the following statement with parameters $b_{0}, b_{1}$, $\mathcal{B}$, and $\mathcal{X}_{0}$ which all lie in $M$ : For each $\delta<\omega_{1}$ there exists an $\alpha \geq \delta$ such that

$$
x_{0}^{\alpha} \cap b_{0} \neq x_{0}^{\beta} \cap b_{0} \quad \text { and } \quad x_{0}^{\alpha} \cap b_{1} \neq x_{0}^{\beta} \cap b_{1} \quad \text { for all } \beta<\delta .
$$

By elementarity this holds in $H_{\mathfrak{c}^{+}}$, so we can recursively construct an uncountable set $\Omega \subseteq \omega_{1}$ such that

$$
x_{0}^{\alpha} \cap b_{0} \neq x_{0}^{\beta} \cap b_{0} \quad \text { and } \quad x_{0}^{\alpha} \cap b_{1} \neq x_{0}^{\beta} \cap b_{1} \quad \text { for all } \alpha \neq \beta \text { in } \Omega .
$$

Since the sequence $\left\{x_{1}^{\alpha}: \alpha \in \omega_{1}\right\}$ is one-to-one, there must be a set $C_{1} \in$ $\left\{b_{0}, b_{1}, \omega \backslash\left(b_{0} \cup b_{1}\right)\right\}$ and an uncountable set $\Omega^{\prime} \subseteq \Omega$ such that

$$
x_{1}^{\alpha} \cap C_{1} \neq x_{1}^{\beta} \cap C_{1} \quad \text { for all } \alpha \neq \beta \text { in } \Omega^{\prime} .
$$


Pick $C_{0} \in\left\{b_{0}, b_{1}\right\}$ to be disjoint from $C_{1}$. Then $\left\langle C_{0}, C_{1}\right\rangle$ separates $\left\{\left\{x_{0}^{\alpha}, x_{1}^{\alpha}\right\}\right.$ : $\left.\alpha \in \Omega^{\prime}\right\}$, contradicting our assumption.

REMARK 3.5. We have just proved the following. Suppose that for some strong antichain $\mathcal{A}$ of $\mathcal{P}$ we cannot find a sequence $\left\langle C_{0}, \ldots, C_{n}\right\rangle$ that separates an uncountable subantichain of $\mathcal{A}$. Then we can obtain an uncountable one-to-one sequence

$$
\mathcal{X}_{0}=\left\{x_{\alpha}:=x_{0}^{\alpha}: \alpha<\omega_{1}\right\}
$$

of elements of $\mathcal{B}$ such that for every countable elementary submodel $M$ of $H_{\mathfrak{c}^{+}}$containing $\mathcal{B}$ and $\mathcal{X}_{0}$, every pair $b_{0}, b_{1}$ of disjoint and nonempty elements of $\mathcal{B} \cap M$, and every $\alpha \geq \delta=M \cap \omega_{1}$, (\#) holds. In other words, we have the conclusion of Lemma 3.1.

Now we proceed further and consider an even stronger form of separation whose failure also leads to an occurrence of a sequence satisfying the conclusion of Lemma 3.1. However, this time we will be able to show that this stronger separation leads to an uncountable subantichain of $\mathcal{B}$. This will clearly complete the proof of Lemma 3.1.

Remark 3.6. Suppose there exist a sequence $\left\langle C_{0}, \ldots, C_{n}\right\rangle$ that separates $\mathcal{A}$ and another sequence $\left\langle D_{0}, \ldots, D_{n}\right\rangle$ of elements of the Boolean algebra $\mathcal{B}$ such that the following holds for every $\alpha \neq \beta<\omega_{1}$ and $i \leq n$ :

(†) $x_{i}^{\alpha} \cap C_{i} \cap D_{i} \neq x_{i}^{\beta} \cap C_{i} \cap D_{i} \quad$ and $\quad C_{i} \backslash\left(D_{i} \cup x_{i}^{\alpha}\right) \neq C_{i} \backslash\left(D_{i} \cup x_{i}^{\beta}\right)$.

Then the elements

$$
B_{\alpha}=\bigcup_{i \leq n}\left(x_{i}^{\alpha} \cap C_{i} \cap D_{i}\right) \cup\left(C_{i} \backslash\left(D_{i} \cup x_{i}^{\alpha}\right)\right),
$$

for $\alpha<\omega_{1}$, form an uncountable antichain of $\mathcal{B}$.

To show this choose $\alpha \neq \beta<\omega_{1}$. Then by Note 3.3 there exists an $i=i_{\alpha \beta} \leq n$ such that $x_{i}^{\alpha} \subseteq x_{i}^{\beta}$ or vice versa. In the former case we have

$$
x_{i}^{\alpha} \cap C_{i} \cap D_{i} \subset x_{i}^{\beta} \cap C_{i} \cap D_{i} \text { and } C_{i} \backslash\left(D_{i} \cup x_{i}^{\beta}\right) \subset C_{i} \backslash\left(D_{i} \cup x_{i}^{\alpha}\right) .
$$

In the latter case the containments reverse. But in both cases $B_{\alpha}$ is incomparable to $B_{\beta}$ since the elements of $\left\langle C_{0}, \ldots, C_{n}\right\rangle$ are pairwise disjoint.

Therefore from now on we can assume that the separation defined in Remark 3.6 is impossible, or in other words that:

(†) for every sequence $\left\langle C_{0}, \ldots, C_{n}\right\rangle$ separating a strong antichain $\mathcal{A}=$ $\left\langle\left\{x_{0}^{\alpha}, \ldots, x_{m_{\alpha}}^{\alpha}\right\}: \alpha<\omega_{1}\right\rangle$ and every tuple $\left\langle D_{0}, \ldots, D_{n}\right\rangle$ of elements of $\mathcal{B}$, there is no uncountable set $\Omega \subseteq \omega_{1}$ such that for all $\alpha \neq \beta$ in $\Omega$ and $i \leq n$ the condition ( $\dagger$ ) holds.

It follows that any attempt to successively pick sets $D_{0}, \ldots, D_{n}$ in $\mathcal{B}$ and uncountable subsets $\Omega_{0} \supseteq \ldots \supseteq \Omega_{n}$ of $\omega_{1}$ in order to satisfy ( $\dagger$ ) for $\alpha \neq \beta$ in $\Omega_{j}$ and $i \leq j \leq n$, must stop at some stage $i_{0}<n$. In other words having 
constructed sets $D_{0}, \ldots, D_{i_{0}-1}$ and uncountable sets $\omega_{1} \supseteq \Omega_{0} \supseteq \ldots \supseteq \Omega_{i_{0}-1}$ such that $(\dagger)$ holds for $\alpha \neq \beta$ in $\Omega_{i_{0}-1}$ and $i<i_{0}$, we will be unable to find a set $D_{i_{0}}$ and an uncountable set $\Omega \subseteq \Omega_{i_{0}-1}$ that would satisfy ( $\dagger$ ) for all $\alpha \neq \beta$ in $\Omega$ and $i=i_{0}$.

From now on let $\mathcal{X}_{0}=\left\{x_{\alpha}:=x_{i_{0}}^{\alpha} \cap C_{i_{0}}: \alpha \in \Omega_{i_{0}-1}\right\}$. Without loss of generality we may work entirely inside the Boolean algebra $\mathcal{B}\left\lceil C_{i_{0}}\right.$. So, in order to simplify notation, we assume $C_{i_{0}}=\omega$, which results in the assumption $i_{0}=n=0$. Moreover, we assume that $\mathcal{B}$ is generated by $\mathcal{X}_{0}$.

Note 3.7. Let $M$ be a countable elementary submodel of $H_{\mathfrak{c}^{+}}$that contains $\mathcal{B}$ and $\mathcal{X}_{0}$. Then for every pair of nonempty disjoint elements $b_{0}, b_{1} \in \mathcal{B} \cap M$ and every $\alpha>\delta=M \cap \omega_{1}$,

$$
x_{\alpha} \cap b_{0} \in M \quad \text { or } \quad x_{\alpha} \cap b_{1} \in M .
$$

In other words, we have reached again the conclusion of Lemma 3.1.

For if this fails for some $\alpha>\delta$ and $b_{0}, b_{1} \in \mathcal{B} \cap M$ then, as before, $M$ would satisfy the following statement with parameters $b_{0}, b_{1}, \mathcal{B}, \mathcal{X}_{0} \in M$ : for all $\delta<\omega_{1}$ there exists an $\alpha>\delta$ such that both $x_{\alpha} \cap b_{0}, x_{\alpha} \cap b_{1} \notin M$. Hence using elementarity this statement is true in $H_{\mathfrak{c}^{+}}$and we can recursively construct an uncountable subsequence of $\mathcal{X}_{0}$ satisfying ( $\dagger$ ) for $i=i_{0}, D_{i_{0}}=b_{0}$, and $C_{i_{0}}=\omega$ or $b_{0} \cup b_{1}$. This contradicts (††). Lemma 3.1 is proved.

4. An equivalence relation on the Stone space. Fix a sequence $\mathcal{X}_{0}=\left\{x_{\alpha}: \alpha \in \omega_{1}\right\}$ of elements of $\mathcal{B}$ satisfying Lemma 3.1. The rest of the paper analyzes the sequence $\mathcal{X}_{0}$ in depth. Let $M$ be a countable elementary submodel of $H_{\mathfrak{c}^{+}}$that contains $\mathcal{B}$ and $\mathcal{X}_{0}$.

Note 4.1. Suppose that for $\alpha<\omega_{1}$, we define the set

$$
\mathcal{I}_{\alpha}^{M}=\left\{b \in \mathcal{B} \cap M: x_{\alpha} \cap b \in M\right\} .
$$

Then for every $\alpha \geq \delta=M \cap \omega_{1}$, the set $\mathcal{I}_{\alpha}^{M}$ is a prime ideal of the Boolean algebra $\mathcal{B} \cap M$. We frequently rely on this observation in what follows.

Denote by $\mathcal{U}$ the Stone space of $\mathcal{B}$ whose elements are all the ultrafilters of $\mathcal{B}$. We define the following equivalence relation on $\mathcal{U}$ (for more on this see $[19])$.

Definition 4.2. If $t, u \in \mathcal{U}$ then $t \equiv_{M} u$ if and only if for every $b$ in $\mathcal{B} \cap M$,

$$
t \in b \text { is equivalent to } u \in b,
$$

where we identify $b$ with the set of all ultrafilters that contain $b$. We denote the equivalence class of $t$ by $[t]$.

The following lemma provides the setting we work in throughout the rest of the paper. 
Lemma 4.3. For each $\alpha \geq \delta$ there exists exactly one equivalence class $[t]$ such that there exist $u_{0}, u_{1} \in[t]$ with $u_{0} \in x_{\alpha}$ but $u_{1} \notin x_{\alpha}$. We denote this equivalence class by $\left[t_{\alpha}\right]$. We say that $x_{\alpha}$ splits $\left[t_{\alpha}\right]$.

Proof. Assume not. First suppose that $x_{\alpha}$ does not split any $[t]$. Then we can express $x_{\alpha}$ as the union of all $[t]$ such that $[t] \subseteq x_{\alpha}$. Let $\left\{b_{n}: n<\omega\right\}$ be a fixed enumeration of $\mathcal{B} \cap M$. By the definition of the equivalence relation, each $[t] \in \mathcal{U} / \equiv_{M}$ can be expressed as $[t]=\bigcap\left\{b_{n}:[t] \subseteq b_{n}\right\}$.

Claim 4.5. For each $[t] \in \mathcal{U} / \equiv_{M}$ such that $[t] \subseteq x_{\alpha}$ there exists an $m \in \omega$ such that $[t] \subseteq b_{m} \subseteq x_{\alpha}$.

Proof. The claim follows from the compactness of the Stone space $\mathcal{U}$. Since $[t]=\bigcap\left\{b_{n}:[t] \subseteq b_{n}\right\}$, we see that its complement, and therefore the complement of $x_{\alpha}$, is contained in the union of the family $\left\{b_{n}^{\mathrm{c}}:[t] \subseteq b_{n}\right\}$ of clopen sets. So there is a finite set $F \subseteq \omega$ such that $[t] \subseteq b_{n}$ for each $n \in F$ and such that

$$
x_{\alpha}^{\mathrm{c}} \subseteq \bigcup_{n \in F} b_{n}^{\mathrm{c}} .
$$

Let $m$ be the index of the element $\bigcap\left\{b_{n}: n \in F\right\}$ of $\mathcal{B} \cap M$. Then $[t] \subseteq b_{m}$ $\subseteq x_{\alpha}$.

Hence we can fix for each $[t] \subseteq x_{\alpha}$ an integer $m([t])$ such that $[t] \subseteq$ $b_{m([t])} \subseteq x_{\alpha}$. In this case

$$
x_{\alpha}=\bigcup\left\{b_{m([t])}:[t] \subseteq x_{\alpha}\right\} .
$$

Since $x_{\alpha}$ is also compact, as a subspace of $\mathcal{U}$, it follows that $x_{\alpha}=\bigcup_{n \in F^{\prime}} b_{n}$, where $F^{\prime}$ is a finite set of integers. Thus $x_{\alpha} \in \mathcal{B} \cap M$, which is a contradiction. Therefore $x_{\alpha}$ must split at least one $[t] \in \mathcal{U} / \equiv_{M}$.

Now assume that $\left[t_{0}\right]$ and $\left[t_{1}\right]$ are two different equivalence classes split by $x_{\alpha}$. Hence there exists an element $b \in \mathcal{B} \cap M$ such that $t_{0} \in b$ but $t_{1} \notin b$. That is, $t_{1} \in b^{\mathrm{c}}$. Since $x_{\alpha} \cap b$ splits $\left[t_{0}\right]$ and $x_{\alpha} \cap b^{\mathrm{c}}$ splits $\left[t_{1}\right]$ and since no member of $\mathcal{B} \cap M$ can split either $\left[t_{0}\right]$ or $\left[t_{1}\right]$, we must have both $x_{\alpha} \cap b$ and $x_{\alpha} \cap b^{\mathrm{c}}$ off $\mathcal{B} \cap M$, contradicting the fact that $\mathcal{I}_{\alpha}^{M}$ is a prime ideal of $\mathcal{B} \cap M$ (see Note 4.1). The lemma follows.

We now distinguish two possibilities.

5. Case 1: Cantor tree. Suppose that there is a countable elementary submodel $M$ of $H_{\mathfrak{c}^{+}}$containing all the relevant objects such that the set of equivalence classes of $\mathcal{U} / \equiv_{M}$ that are split is uncountable, i.e., there is an uncountable set $\Omega \subseteq \omega_{1}$ such that $\mathcal{X}_{\Omega}=\left\{x_{\alpha}: \alpha \in \Omega\right\} \subseteq \mathcal{X}_{0} \backslash M$ satisfies $\left[t_{\alpha}\right] \neq\left[t_{\beta}\right]$ whenever $\alpha \neq \beta \in \Omega$.

Definition 5.1. Let $x_{\alpha}, x_{\beta} \in \mathcal{X}_{\Omega}$. Recall that $x_{\alpha}$ and $x_{\beta}$ are subsets of $\omega$, so it is natural to define $x_{\alpha}<_{\operatorname{lex}} x_{\beta}$ to mean that there exists an $n_{0}<\omega$ 
such that for $m<n_{0}$,

$$
m \in x_{\alpha} \quad \text { if and only if } m \in x_{\beta}
$$

but

$$
n_{0} \in x_{\beta} \backslash x_{\alpha} .
$$

Definition 5.2. Let $\mathcal{Q}$ be the poset of all finite $F \subseteq \mathcal{X}_{\Omega}$ such that for all $x_{\alpha} \neq x_{\beta} \in F$,

$$
x_{\alpha}<_{\text {lex }} x_{\beta} \text { implies }\left[t_{\alpha}\right] \subseteq x_{\beta} .
$$

Let $\mathcal{R}$ be the poset of all finite $F \subseteq \mathcal{X}_{\Omega}$ such that for all $x_{\alpha} \neq x_{\beta} \in F$,

$$
x_{\alpha}<_{\operatorname{lex}} x_{\beta} \text { implies }\left[t_{\beta}\right] \nsubseteq x_{\alpha} .
$$

REMARK 5.3. The reason for considering these posets is that we will prove, using $\mathrm{MA}_{\omega_{1}}$, that if $\mathcal{Q}$ and $\mathcal{R}$ are both c.c.c. then there is an uncountable chain $Y$ in the power set algebra of some set $S$, such that if $\langle Y\rangle$ is the subalgebra generated by $Y$, then incomparable elements in $\langle Y\rangle$ correspond to incomparable elements in $\mathcal{B}$. Thereby the problem is reduced to Lemma 2.8. On the other hand, Lemmas 5.5 and 5.7 show that if one of $\mathcal{Q}$ or $\mathcal{R}$ is not c.c.c. then $\mathcal{B}$ also has an uncountable family of pairwise incomparable sets. Hence, in general, under the assumptions of Case 1 we get a positive answer to Question 2.1.

Lemma 5.4. $\left(\mathrm{MA}_{\omega_{1}}\right)$ If $\mathcal{Q}$ and $\mathcal{R}$ are c.c.c. then $\mathcal{B}$ has an uncountable antichain.

Proof. Applying $\mathrm{MA}_{\omega_{1}}$ successively to $\mathcal{Q}$ and then $\mathcal{R}$ we obtain an uncountable set $\Gamma \subseteq \Omega$ such that for $\alpha, \beta \in \Gamma$,

$$
x_{\alpha}<_{\operatorname{lex}} x_{\beta} \text { implies }\left[t_{\alpha}\right] \subseteq x_{\beta} \text { and }\left[t_{\beta}\right] \nsubseteq x_{\alpha} .
$$

Let $S=\bigcup_{\alpha \in \Gamma}\left[t_{\alpha}\right]$. Then for $\alpha, \beta \in \Gamma, x_{\alpha}<_{\operatorname{lex}} x_{\beta}$ implies $x_{\alpha} \cap S \subset x_{\beta} \cap S$. Let $Y=\left\{x_{\alpha} \cap S: \alpha \in \Gamma\right\}$. Then $Y$ is a chain in the Boolean algebra $\mathcal{P}(S)$. Furthermore, by Lemma 2.8 the Boolean algebra $\langle Y\rangle$ generated by $Y$ has an uncountable antichain. Note that

$$
\langle Y\rangle=\left\{x \cap\langle S\rangle: x \in\left\langle\left\{x_{\alpha}: \alpha \in \Gamma\right\}\right\rangle\right\} .
$$

So an uncountable antichain of $\langle Y\rangle$ leads to an uncountable antichain of $\left\langle\left\{x_{\alpha}: \alpha \in \Gamma\right\}\right\rangle$ and therefore one of $\mathcal{B}$.

Lemma 5.5. If $\mathcal{Q}$ is not c.c.c. then $\mathcal{B}$ has an uncountable antichain.

Proof. Assume that $\mathcal{Q}$ is not c.c.c. Then $\mathcal{Q}$ has an uncountable strong antichain $\mathcal{A}^{\prime}=\left\langle q_{\alpha}: \alpha<\omega_{1}\right\rangle$. Using a standard $\Delta$-system argument, we may assume that there is an $n^{\prime}<\omega$ such that for all $\alpha<\omega_{1}$,

$$
q_{\alpha}=\left\{x_{i}^{\alpha}: i \leq n^{\prime}\right\}
$$

is listed according to $<_{\text {lex }}$ and $q_{\alpha} \cap q_{\beta}=\emptyset$ for $\alpha \neq \beta<\omega_{1}$. 
Here $x_{i}^{\alpha}$ splits $\left[t_{i}^{\alpha}\right]$ for $i \leq n^{\prime}$.

Recall that $\mathcal{B} \cap M$ is countable and that $\left\langle b_{n}: n \in \omega\right\rangle$ is a fixed enumeration of the elements of $\mathcal{B} \cap M$. Hence we may assume there exist a $k<\omega$ and distinct elements $s_{i} \in\{0,1\}^{k}$ for $i \leq n^{\prime}$ such that

(1) For each $\alpha<\omega_{1}, i \leq n^{\prime}$, and $j<k$,

$$
\left[t_{i}^{\alpha}\right] \subseteq b_{j} \quad \text { if and only if } \quad s_{i}(j)=1 .
$$

(2) The sets $C_{i}^{\prime}=\bigcap\left\{b_{j}: j<k, s_{i}(j)=1\right\}\left(i \leq n^{\prime}\right)$ are disjoint and they separate the elements split by $q_{\alpha}$ for all $\alpha<\omega_{1}$ (i.e., $\left[t_{i}^{\alpha}\right] \subseteq C_{i}^{\prime}$ for all $i \leq n^{\prime}$ and $\left.\alpha<\omega_{1}\right)$.

For $i \leq n^{\prime}$ and $\alpha<\omega_{1}$ define $C_{i}^{\alpha}$ to be the minimal element of the Boolean algebra generated by $\left\{x_{j}^{\alpha}: i \neq j \leq n^{\prime}\right\} \cup\left\{C_{i}^{\prime}\right\}$ that contains $\left[t_{i}^{\alpha}\right]$. Note that by (2) the elements $C_{0}^{\alpha}, \ldots, C_{n^{\prime}}^{\alpha}$ are pairwise disjoint. Since for each $j \leq n^{\prime}, j \neq i$, the element $x_{j}^{\alpha} \cap C_{i}^{\prime}$ is in $\mathcal{B} \cap M$ it follows that $C_{i}^{\alpha}$ is also in $\mathcal{B} \cap M$. Since $\mathcal{B} \cap M$ is countable, we may assume by further refining the strong antichain that for some fixed sequence $C_{0}^{*}, \ldots, C_{n^{\prime}}^{*}$ we have $C_{i}^{\alpha}=C_{i}^{*}$ for all $\alpha<\omega_{1}$ and $i \leq n^{\prime}$. Note that this, in particular, means that $C_{i}^{*} \subseteq x_{j}^{\alpha}$ for all $0 \leq i<j \leq n^{\prime}$ and $\alpha<\omega_{1}$.

Thus for $\alpha \neq \beta<\omega_{1}$,

(+) there is an $i=i_{\alpha, \beta} \leq n^{\prime}$ such that $x_{i}^{\alpha}<_{\operatorname{lex}} x_{i}^{\beta}$ but $\left[t_{i}^{\alpha}\right] \not x_{i}^{\beta}$, or vice versa (with the roles of $\alpha$ and $\beta$ interchanged).

Refining even more, if necessary, we may assume that for $i \leq n^{\prime}$ and $\alpha \neq \beta<\omega_{1}$,

$$
x_{i}^{\alpha} \cap\left(\omega \backslash C_{i}^{*}\right)=x_{i}^{\beta} \cap\left(\omega \backslash C_{i}^{*}\right) .
$$

Hence $x_{i}^{\alpha} \cap C_{i}^{*}<_{\operatorname{lex}} x_{i}^{\beta} \cap C_{i}^{*}$ if and only if $x_{i}^{\alpha}<_{\operatorname{lex}} x_{i}^{\beta}$.

For $\alpha<\omega_{1}$, define

$$
d_{\alpha}=\bigcup_{i \leq n^{\prime}}\left(x_{i}^{\alpha} \cap C_{i}^{*}\right) .
$$

Fix any $\alpha \neq \beta<\omega_{1}$. The lemma follows from

Claim 5.6. $d_{\alpha}$ is incomparable to $d_{\beta}$.

Proof. By $(+)$ we may assume without loss of generality that there is an $i \leq n^{\prime}$ such that $x_{i}^{\alpha}<_{\operatorname{lex}} x_{i}^{\beta}$ but $\left[t_{i}^{\alpha}\right] \not \subseteq x_{i}^{\beta}$. This implies that $\left[t_{i}^{\alpha}\right] \cap x_{i}^{\beta}=\emptyset$, which means that $x_{i}^{\alpha} \backslash x_{i}^{\beta} \neq \emptyset$ as witnessed by $\left[t_{i}^{\alpha}\right]$. Thus $\left(x_{i}^{\alpha} \cap C_{i}^{*}\right) \backslash\left(x_{i}^{\beta} \cap\right.$ $\left.C_{i}^{*}\right) \neq \emptyset$. On the other hand, since $x_{i}^{\alpha} \cap C_{i}^{*}<_{\operatorname{lex}} x_{i}^{\beta} \cap C_{i}^{*}$ if and only if $x_{i}^{\alpha}<_{\text {lex }} x_{i}^{\beta}$, by definition we have $\left(x_{i}^{\beta} \cap C_{i}^{*}\right) \backslash\left(x_{i}^{\alpha} \cap C_{i}^{*}\right) \neq \emptyset$.

Hence $x_{i}^{\alpha} \cap C_{i}^{*}$ is incomparable to $x_{i}^{\beta} \cap C_{i}^{*}$. Since the elements of $\left\langle C_{0}^{*}, \ldots\right.$ $\left.\ldots, C_{n^{\prime}}^{*}\right\rangle$ are pairwise disjoint, $d_{\alpha}$ is incomparable to $d_{\beta}$ as desired. This shows Claim 5.6 and hence also Lemma 5.5. 
Similarly one can prove

Lemma 5.7. If $\mathcal{R}$ is not c.c.c. then $\mathcal{B}$ has an uncountable antichain.

Proof. Keeping the same notation as above, we separate an arbitrary strong antichain $\mathcal{A}^{\prime}$ of $\mathcal{R}$ with $\left\langle C_{0}^{*}, \ldots, C_{n^{\prime}}^{*}\right\rangle$. Note that in this case, $x_{i}^{\alpha} \cap C_{j}^{*}$ $=\emptyset$ for all $0 \leq i<j \leq n^{\prime}$ and $\alpha<\omega_{1}$.

Thus we may also assume that for $\alpha \neq \beta<\omega_{1}$,

$(++) \quad$ there is an $i=i_{\alpha, \beta} \leq n^{\prime}$ such that $x_{i}^{\alpha}<_{\operatorname{lex}} x_{i}^{\beta}$ but $\left[t_{i}^{\beta}\right] \subseteq x_{i}^{\alpha}$, or vice versa (with the roles of $\alpha$ and $\beta$ interchanged),

and, similarly, that for $i \leq n^{\prime}$ and $\alpha \neq \beta<\omega_{1}$,

$$
x_{i}^{\alpha} \cap\left(\omega \backslash C_{i}^{*}\right)=x_{i}^{\beta} \cap\left(\omega \backslash C_{i}^{*}\right) .
$$

Hence again $x_{i}^{\alpha} \cap C_{i}^{*}<_{\operatorname{lex}} x_{i}^{\beta} \cap C_{i}^{*}$ if and only if $x_{i}^{\alpha}<_{\operatorname{lex}} x_{i}^{\beta}$. Once again for $\alpha<\omega_{1}$, define

$$
d_{\alpha}=\bigcup_{i<n^{\prime}}\left(x_{i}^{\alpha} \cap C_{i}^{*}\right)
$$

As before, it remains to prove the following claim.

Claim 5.8. $d_{\alpha}$ is incomparable to $d_{\beta}$ for $\alpha \neq \beta<\omega_{1}$.

Proof. By $(++)$ there is an $i \leq n^{\prime}$ such that $x_{i}^{\alpha}<_{\operatorname{lex}} x_{i}^{\beta}$ but $\left[t_{i}^{\beta}\right] \subseteq x_{i}^{\alpha}$. As in the previous proof, in this case it is also true that $x_{i}^{\alpha} \backslash x_{i}^{\beta} \neq \emptyset$, although here the fact is witnessed by $\left[t_{i}^{\beta}\right]$. Thus $\left(x_{i}^{\alpha} \cap C_{i}^{*}\right) \backslash\left(x_{i}^{\beta} \cap C_{i}^{*}\right) \neq \emptyset$. On the other hand, since $x_{i}^{\alpha} \cap C_{i}^{*}<_{\operatorname{lex}} x_{i}^{\beta} \cap C_{i}^{*}$ if and only if $x_{i}^{\alpha}<_{\operatorname{lex}} x_{i}^{\beta}$, by the definition of $<_{\text {lex }}$ we have $\left(x_{i}^{\beta} \cap C_{i}^{*}\right) \backslash\left(x_{i}^{\alpha} \cap C_{i}^{*}\right) \neq \emptyset$. This proves Claim 5.8 and hence Lemma 5.7.

This finishes our analysis of Case 1.

6. Case 2: Aronszajn tree. The set of equivalence classes of $\mathcal{U} / \equiv_{M}$ that are split by elements of the set $\mathcal{X}_{0}=\left\{x_{\alpha}: \alpha<\omega_{1}\right\}$ of Lemma 3.1 is countable for any countable elementary submodel $M$ of $H_{\mathfrak{c}^{+}}$.

Let $\left\langle M_{\alpha}: \alpha<\omega_{1}\right\rangle$ be a continuous $\in$-chain of countable elementary submodels of $\left(H_{\mathfrak{c}^{+}}, \in\right)$. Assume that $\mathcal{B}, \mathcal{X}_{0} \in M_{0}$. For $\alpha<\omega_{1}$, let $\mathcal{B}_{\alpha}=$ $\mathcal{B} \cap M_{\alpha}$. Let

$$
\Omega=\left\{\alpha \in \omega_{1}: M_{\alpha} \cap \alpha=\alpha\right\} .
$$

Then $\Omega$ is a closed unbounded subset of $\omega_{1}$. Note that for $\alpha \in \Omega, x_{\alpha} \notin \mathcal{B}_{\alpha}$ but there exists $\beta \in \Omega$ with $x_{\alpha} \in \mathcal{B}_{\beta}$.

Definition 6.1. For $\alpha \in \Omega$ we define $\left[t_{\alpha}\right]$ as follows. Let $\equiv_{\alpha}$ be the equivalence relation on the Stone space induced by $\mathcal{B}_{\alpha}$ as defined above in Definition 4.2. Then $\left[t_{\alpha}\right]$ is the equivalence class of $\equiv_{\alpha}$ split by $x_{\alpha}$. 
Note 6.2. Let $A$ be the set of all split equivalence classes (by members of the set $\mathcal{X}_{0}$ ) induced by the equivalence relation $\equiv_{\alpha}$ for some $\alpha \in \Omega$. Let $A_{\alpha}$ denote the set of split equivalence classes of $\equiv_{\alpha}$. Note that the hypotheses of Case 2 imply that each $A_{\alpha}$ is countable. Note also that $A$ does not have an uncountable chain since a strictly decreasing $\omega_{1}$-sequence of closed sets of $\mathcal{U}$ (elements of $A$ ) easily gives an uncountable $\mathcal{Y} \subseteq \mathcal{B}$ which is well-founded under $\subseteq$ and hence, by Lemma $2.5, \mathcal{B}$ would have an uncountable antichain. It follows that $A$ is an Aronszajn tree ordered under reverse inclusion. Let $S=\left\{\left[t_{\alpha}\right]: \alpha \in \Omega\right\} \subseteq A$. Going to an uncountable (or even stationary) subset of $\Omega$, we may assume (cf. [15, 9.5] or Lemma 6.5 below) that $S$ is an antichain of $A$. Hence for $\alpha<\beta$ in $\Omega, x_{\beta}$ does not split $\left[t_{\alpha}\right]$ (and vice versa, $x_{\alpha}$ does not split $\left.\left[t_{\beta}\right]\right)$.

Notation 6.3. Let $\gamma \leq \beta<\omega_{1}$. We denote by $\left[x_{\beta}\right]_{\gamma}$ the equivalence class of $\equiv_{\gamma}$ split by $x_{\beta}$. Note that in this notation, $\left[t_{\beta}\right]=\left[x_{\beta}\right]_{\beta}$.

Remark 6.4. Let $\alpha \neq \beta$ be members of $\Omega$ and let $\gamma=\min \{\alpha, \beta\}$. Then $\left[x_{\alpha}\right]_{\gamma} \neq\left[x_{\beta}\right]_{\gamma}$. This means that either $\left[x_{\alpha}\right]_{\gamma} \subseteq x_{\beta}$ or $\left[x_{\alpha}\right]_{\gamma} \cap x_{\beta}=\emptyset$. In particular, for all $[s] \in A$ included in $\left[x_{\alpha}\right]_{\gamma}$ we have $[s] \subseteq x_{\beta}$ if and only if $\left[x_{\alpha}\right]_{\gamma} \subseteq x_{\beta}$. This observation is used repeatedly (and implicitly) below.

We shall rely frequently on the following well-known result about Aronszajn trees (see [15, Chapters 5 and 9] for more details related to this fact).

Lemma 6.5. $\left(\mathrm{MA}_{\omega_{1}}\right)$ Suppose $T$ is an Aronszajn tree and $\mathcal{F}$ is an uncountable family of pairwise disjoint finite subsets of $T$. Then there is an uncountable subfamily $\mathcal{F}_{0}$ such that for every pair $F$ and $G$ of distinct elements of $\mathcal{F}_{0}$, every node of $F$ is incomparable with every node of $G$.

P r o of. Clearly, we may assume that elements of $\mathcal{F}$ are all of the same fixed size $n+1$ and that for each $F \in \mathcal{F}$ we have fixed its enumeration $\left\{x_{0}^{F}, \ldots, x_{n}^{F}\right\}$. By $\mathrm{MA}_{\omega_{1}}, T$ contains no Suslin subtree, or equivalently, every uncountable subset of $T$ contains an uncountable antichain. Applying this successively $n+1$ times, and going to an uncountable subfamily of $\mathcal{F}$, we may assume that for each $i \leq n$, the set $\left\{x_{i}^{F}: F \in \mathcal{F}\right\}$ is an antichain of $T$. Refining $\mathcal{F}$ further we may assume we have a natural well-ordering $\preceq$ of $\mathcal{F}$ respecting the heights in $T$, i.e., $F \prec G$ in $\mathcal{F}$ implies that every node of $F$ is of smaller height than any node of $G$. For $i, j \leq n$, let $\mathcal{K}_{i, j}$ be the collection of all pairs $\{F, G\}$ of elements of $\mathcal{F}$ such that $F \prec G$ implies that $x_{i}^{F}<_{T} x_{j}^{G}$. Applying the Dushnik-Miller partition theorem ([6]), either we can find an uncountable $\mathcal{F}_{0} \subseteq \mathcal{F}$ with no pair belonging to any set $\mathcal{K}_{i, j}$ or there exist three elements $F \prec G \prec H$ of $\mathcal{F}$ and a pair of coordinates $i, j \leq n$ such that

$$
\{F, H\},\{G, H\} \in \mathcal{K}_{i, j} .
$$

Thus $x_{i}^{F}$ and $x_{i}^{G}$ are both predecessors of $x_{j}^{H}$ and are therefore comparable, 
contradicting our initial assumption that the $i$ th projection of the family $\mathcal{F}$ is an antichain of $T$. This shows that the first alternative given by the Dushnik-Miller theorem must take place, giving us the conclusion of the lemma.

We shall also work extensively with lexicographical orderings of the Aronszajn tree $A$. In what follows we include some of the definitions. The reader is referred to [15] for more details about this.

Definition 6.6. For a given tree $T$, let $P(T)$ denote the collection of all paths of $T$, i.e., chains of $T$ which are downwards closed. For example, if $u$ and $v$ are nodes of $T$ then $p(u, v)$ is the path of all $t \in T$ such that $t \leq_{T} u$ and $t \leq_{T} v$. For a given $p \in P(T)$ let $N_{p}$ be the collection of all $u \in T$ such that $t<_{T} u$ for all $t \in p$ but no $v<_{T} u$ has this property. Thus $N_{p}$ is the collection of all "immediate successors of $p$ ". A lexicographical ordering $<_{\text {Lex }}$ of $T$ is determined by the collection $\left(N_{p},<_{p}\right)(p \in P(T))$ of total orderings as follows: $s<_{\text {Lex }} t$ if and only if $s<_{T} t$ or $s$ and $t$ are incomparable and $u<_{p} v$ where $p=p(s, t)$ and where $u$ is the unique element of $N_{p}$ that precedes $s$ and where $v$ is the unique element $N_{p}$ that precedes $t$.

Note 6.7. It has been shown that every Countryman ordering (see Definition 2.2) is isomorphic to a lexicographically ordered Aronszajn tree and that there exist Aronszajn trees with lexicographical orderings that are Countryman (see [12], [15], [18]).

The following lemma describes the typical behaviour of uncountable sequences of finite subsets of some lexicographically ordered Aronszajn tree.

Lemma 6.8. Suppose $T$ is a given Aronszajn tree with a fixed lexicographical ordering $<_{\mathrm{Lex}}$. Then for every positive integer $n$ and every $\omega_{1}$ sequence $\left\langle\left\{t_{1}^{\alpha}, \ldots, t_{n}^{\alpha}\right\}: \alpha<\omega_{1}\right\rangle$ of pairwise disjoint n-element subsets of $T$ listed according to $<_{\mathrm{Lex}}$, there exist an uncountable set $\Gamma \subseteq \omega_{1}$, an integer $0<k \leq n$, a sequence of integers $s_{0}, s_{1}, \ldots, s_{k}$ with $s_{0}=0$, a one-to-one sequence $r_{1}, \ldots, r_{k}$ of elements of some fixed level $T_{\gamma_{0}}$ of $T$, and for each $\gamma \in \Gamma$ a sequence $r_{1}^{\gamma}, \ldots, r_{k}^{\gamma}$ of elements of $T_{\gamma}$ such that

(a) $s_{0}+s_{1}+\ldots+s_{k}=n$

(b) $r_{i}^{\gamma}$ extends $r_{i}$ for all $\gamma \in \Gamma$ and $i \in\{1, \ldots, k\}$,

(c) $t_{i}^{\gamma}$ extends $r_{j}^{\gamma}$ whenever $\gamma \in \Gamma, j<k$ and $s_{0}+\ldots+s_{j}<i \leq$ $s_{0}+\ldots+s_{j+1}$.

Proof. Re-enumerating the sequence we may assume that $t_{i}^{\alpha}$ has height greater than or equal to $\alpha$ for all $\alpha<\omega_{1}$ and $i=1, \ldots, k$. So let $\left\{r_{j}^{\alpha}: 1 \leq j \leq\right.$ $\left.k_{\alpha}\right\}$ enumerate according to $<_{\text {Lex }}$ the projection of the set $\left\{t_{i}^{\alpha}: 1 \leq i \leq n\right\}$ onto the $\alpha$ th level of $T$. Then there is a stationary set $\Delta \subseteq \omega_{1}$ and an integer $k$ such that $k_{\alpha}=k$ for all $\alpha$. For a limit ordinal $\gamma \in \Delta$ let $f(\gamma)<\gamma$ be some 
ordinal with the property that the projection of $\left\{r_{j}^{\gamma}: 1 \leq j \leq k\right\}$ onto $T_{f(\gamma)}$ is one-to-one. Find a stationary set $\Gamma \subseteq \Delta$ and an ordinal $\gamma_{0}<\omega_{1}$ such that $f(\gamma)=\gamma_{0}$ for all $\gamma \in \Gamma$ and such that for some $r_{1}, \ldots, r_{k} \in T_{\gamma_{0}}$ the projection of $r_{j}^{\gamma}$ onto $T_{\gamma_{0}}$ is equal to $r_{j}$ for all $\gamma \in \Gamma$ and $j=1, \ldots, k$. Note that by the definition of a lexicographical ordering, the enumeration $r_{1}, \ldots, r_{k}$ respects $<_{\text {Lex }}$ since for every $\gamma \in \Gamma$ and $1 \leq j, l \leq k$ the relation $r_{j}^{\gamma}<$ Lex $r_{l}^{\gamma}$ is determined at the same splitting node where the relation $r_{j}<_{\text {Lex }} r_{l}$ is determined. Note also that for a fixed $\gamma \in \Gamma$ the sets

$$
I_{j}^{\gamma}=\left\{i \in\{1, \ldots, n\}: t_{i}^{\gamma} \text { extends } r_{j}\right\} \quad(1 \leq j \leq k)
$$

are intervals of integers with the property that $I_{j}^{\gamma}$ lies below $I_{l}^{\gamma}$ whenever $1 \leq j<l \leq k$. Let $s_{j}^{\gamma}=\left|I_{j}^{\gamma}\right|$ for $j=1, \ldots, k$. Shrinking $\Gamma$ further we may assume that for some fixed sequence of integers $s_{1}, \ldots, s_{k}$ we have $s_{j}^{\gamma}=s_{j}$ for all $\gamma \in \Gamma$ and $1 \leq j \leq k$. It should be clear that the objects thus obtained satisfy the conditions (a), (b) and (c) above.

From now on we consider a fixed lexicographic order $<_{\text {Lex }}$ on the tree of all split equivalence classes $A$. It will be useful to assume that $\left(A,<_{\text {Lex }}\right)$ is a Countryman order. We will justify our assumption below in Lemma 6.16.

Definition 6.9. Let $\mathcal{Q}=\mathcal{Q}\left\langle\left[t_{\alpha}\right], x_{\alpha}: \alpha \in \Omega\right\rangle$ be the partial order of all finite subsets $q=\left\{\left[t^{1}\right], \ldots,\left[t^{m}\right]\right\}$ of $S$ such that for $0<i, j \leq m$,

$$
\left[t^{i}\right]<_{\text {Lex }}\left[t^{j}\right] \text { implies }\left[t^{i}\right] \subseteq x^{j},
$$

where for $0<j \leq m, x^{j}$ is the corresponding element of $\mathcal{X}_{0}$ that splits $\left[t^{j}\right]$ (i.e., if $\left[t^{j}\right]=\left[t_{\alpha}\right]$ for some $\alpha \in \Omega$ then $x^{j}$ is the element $x_{\alpha}$ of $\mathcal{X}_{0}$ ).

Note 6.10 . We define the partial order $\mathcal{Q}$ depending on the parameter $\left\langle\left[t_{\alpha}\right], x_{\alpha}: \alpha \in \Omega\right\rangle$ since we may need to replace it with any other parameter $\left\langle\left[r_{\alpha}\right], y_{\alpha}: \alpha \in \Omega_{1}\right\rangle$, where

(1) $\Omega_{1}$ is an uncountable subset of $\Omega$,

(2) $\left[r_{\alpha}\right]$ is a split equivalence class of $\equiv_{\alpha}$,

(3) $y_{\alpha}$ is a member of $\mathcal{B}$ which splits $\left[r_{\alpha}\right]$ but not any other class of $\equiv_{\alpha}$,

(4) $\left\langle\left[r_{\alpha}\right]: \alpha \in \Omega_{1}\right\rangle$ is an antichain of the tree $A$.

The posets of the form $\mathcal{Q}\left\langle\left[r_{\alpha}\right], y_{\alpha}: \alpha \in \Omega_{1}\right\rangle$ for some parameter $\left\langle\left[r_{\alpha}\right], y_{\alpha}\right.$ : $\left.\alpha \in \Omega_{1}\right\rangle$ satisfying (1)-(4) will be called versions of $\mathcal{Q}$.

We now show that regardless of whether $\mathcal{Q}$ is c.c.c. or not, there is an uncountable subset $\mathcal{Y}$ of $(\mathcal{B}, \subseteq)$ and a strictly increasing map from $(\mathcal{Y}, \subseteq)$ into a Countryman order. Hence Lemma 2.5 implies that in both cases we obtain an uncountable antichain of $\mathcal{B}$.

Lemma 6.11. If $\mathcal{Q}$ is not c.c.c. then an uncountable subset of $(\mathcal{B}, \subseteq)$ can be extended to a Countryman order. 
Proof. Assume $\mathcal{Q}$ is not c.c.c. Let $R=\left\langle q_{\alpha}=\left\{\left[t_{1}^{\alpha}\right], \ldots,\left[t_{m_{\alpha}}^{\alpha}\right]\right\}: \alpha<\omega_{1}\right\rangle$ be an uncountable sequence of pairwise incompatible elements of $\mathcal{Q}$. Using a $\Delta$-system argument we may assume that the elements of $R$ are pairwise disjoint and there exists an $n<\omega$ such that $m_{\alpha}=n$ for all $\alpha<\omega_{1}$.

Using Lemma 6.8 and refining still further, we may assume there exist an integer $k \leq n$, a $\gamma \in \omega_{1}$, elements $r_{1}, \ldots r_{k} \in A_{\gamma}$ and integers $s_{0}, \ldots, s_{k} \in \omega$ with $s_{0}=0$ and $s_{0}+\ldots+s_{k}=n$ such that:

(1) If $j<k$ and $s_{0}+\ldots+s_{j}<i \leq s_{0}+\ldots+s_{j+1}$ then $\left[t_{i}^{\alpha}\right]$ extends $r_{j+1}$.

(2) For each $\alpha<\omega_{1}$ there exist elements $r_{1}^{\alpha}, \ldots r_{k}^{\alpha} \in A$ such that for $j<k, r_{j+1}^{\alpha}$ extends $r_{j+1}$ and $\left[t_{i}^{\alpha}\right]$ extends $r_{j+1}^{\alpha}$ for all $s^{j}<i \leq s^{j+1}$, where $s^{j}=s_{0}+\ldots+s_{j}$. If for some $0<j \leq k$ we have $s_{j}=1$ then we take $r_{j}^{\alpha}=\left[t_{s^{j}}^{\alpha}\right]$.

(3) For each $\alpha<\omega_{1}$ and $0<j \leq k$, the height of $r_{j}^{\alpha}$ in the tree is $\geq \alpha$.

Applying Lemma 6.5 we may further assume that in $A$.

(4) For each $\alpha \neq \beta<\omega_{1}$ and $0<i, j \leq k, r_{i}^{\alpha}$ and $r_{j}^{\beta}$ are incomparable

Before we proceed, let us explain the scenario of the rest of the proof. We show below that the fact that $R$ is a strong antichain implies that for $\alpha \neq \beta<\omega_{1}$ there exists an $i=i_{\alpha, \beta}$ such that

$$
\left[t_{i}^{\alpha}\right]<_{\text {Lex }}\left[t_{i}^{\beta}\right] \text { but }\left[t_{i}^{\alpha}\right] \nsubseteq x_{i}^{\beta},
$$

which in turn implies that $x_{i}^{\alpha} \nsubseteq x_{i}^{\beta}$. In other words, $x_{i}^{\alpha} \subseteq x_{i}^{\beta}$ implies $\left[t_{i}^{\beta}\right]<_{\text {Lex }}\left[t_{i}^{\alpha}\right]$. (Recall that $x_{i}^{\alpha}$ is our notation for the associated element of $\mathcal{X}_{0}$ that splits $\left[t_{i}^{\alpha}\right]$.)

As with the posets in Case 1, we take advantage of this fact by separating $R$ with a suitable sequence $\left\langle C_{1}, \ldots, C_{n}\right\rangle$ of elements of $\mathcal{B}$. The difference in this case will be that we do not need the separating sequence $\left\langle C_{1}, \ldots, C_{n}\right\rangle$ to obtain $(+)$.

On the other hand, since we have assumed that $A$ with the ordering $<_{\text {Lex }}$ is a Countryman ordering then we may also assume that $R$ is a chain in $A^{n}$ when we replace its elements by the $n$-tuples that enumerate them. Therefore for $\alpha \neq \beta<\omega_{1}$,

$$
x_{i}^{\alpha} \subseteq x_{i}^{\beta} \quad \text { implies that, in fact, }\left[t_{1}^{\beta}\right]<_{\text {Lex }}\left[t_{1}^{\alpha}\right] .
$$

Define, for $\alpha<\omega_{1}$,

$$
a_{\alpha}=\bigcup_{0<i \leq n}\left(x_{i}^{\alpha} \cap C_{i}\right) .
$$

Then whenever $\alpha \neq \beta<\omega_{1}$ the inclusion $a_{\alpha} \subseteq a_{\beta}$ implies that $\left[t_{1}^{\beta}\right]<_{\text {Lex }}\left[t_{1}^{\alpha}\right]$. Hence an uncountable subset of $\mathcal{B}$ is extended by the lexicographic order 
on $A$, which is also a Countryman order. This reduces the case to Lemma 2.5. The particulars follow.

Claim 6.12. For $\alpha \neq \beta<\omega_{1}$ there exists an $i=i_{\alpha, \beta}$ such that $\left[t_{i}^{\alpha}\right]<_{\text {Lex }}$ $\left[t_{i}^{\beta}\right]$ but $\left[t_{i}^{\alpha}\right] \nsubseteq x_{i}^{\beta}$.

Proof. Since $R$ is a strong antichain, for $\alpha \neq \beta<\omega_{1}$ there exist $0<$ $i, j \leq n$ such that

$$
\left[t_{i}^{\alpha}\right]<_{\text {Lex }}\left[t_{j}^{\beta}\right] \text { but }\left[t_{i}^{\alpha}\right] \nsubseteq x_{j}^{\beta} .
$$

In particular, this means that $x_{j}^{\beta} \cap\left[t_{i}^{\alpha}\right]=\emptyset$.

If there are $0<l_{0} \neq l_{1} \leq k$ such that $\left[t_{i}^{\alpha}\right]$ extends $r_{l_{0}}$ and $\left[t_{j}^{\beta}\right]$ extends $r_{l_{1}}$, then $\left[t_{i}^{\beta}\right]$ also extends $r_{l_{0}}$. By assumption $x_{j}^{\beta} \cap\left[t_{i}^{\alpha}\right]=\emptyset$, hence by Remark 6.4 $x_{j}^{\beta} \cap r_{l_{0}}=\emptyset$. Therefore $x_{j}^{\beta} \cap\left[t_{i}^{\beta}\right]=\emptyset$, which is a contradiction.

So let $0<l \leq k$ be such that both $\left[t_{i}^{\alpha}\right]$ and $\left[t_{j}^{\beta}\right]$ extend $r_{l}$. Using Remark 6.4 again we see that $x_{j}^{\beta} \cap\left[t_{i}^{\alpha}\right]=\emptyset$ implies $x_{j}^{\beta} \cap r_{l}^{\alpha}=\emptyset$. In particular, it follows that $x_{j}^{\beta} \cap\left[t_{j}^{\alpha}\right]=\emptyset$. Thus we may assume that $i=j$.

We now argue that the general case can be reduced to the case $k=n$. To show this we associate with each element of $R$ an element of some other version of $\mathcal{Q}$ of size $k$ which effectively represents it as a member of a strong antichain in that version of $\mathcal{Q}$.

For $\alpha<\omega_{1}$, let $q_{\alpha}^{\prime}=\left\{r_{1}^{\alpha}, \ldots, r_{k}^{\alpha}\right\}$ be the element that corresponds to $q_{\alpha} \in R$. With each $r_{j+1}^{\alpha} \in A$ with $\alpha<\omega_{1}$ and $j<k$, we associate the element

$$
X_{j+1}^{\alpha}=\bigcap_{s^{j}<i \leq s^{j+1}} x_{i}^{\alpha} \in \mathcal{B},
$$

where as above $s^{j}=s_{0}+\ldots+s_{j}$.

In particular, if $s_{j+1}=1$ then $X_{j+1}^{\alpha}=x_{s^{j+1}}^{\alpha}$.

REMARK 6.13. Note that since $x_{i}^{\alpha}$ splits $r_{j+1}^{\alpha}$ for all $s^{j}<i \leq s^{j+1}$, it follows by Remark 6.4 that if $X_{j+1}^{\alpha}$ splits any equivalence class at the level of $r_{j+1}^{\alpha}$ at all, it must split $r_{j+1}^{\alpha}$. Thus $X_{j+1}^{\alpha}$ splits $r_{j+1}^{\alpha}$ if $X_{j+1}^{\alpha} \cap r_{j+1}^{\alpha} \neq \emptyset$. If $s_{j+1}=1$ then by assumption $X_{j+1}^{\alpha}=x_{s^{j+1}}^{\alpha}$ splits $r_{j+1}^{\alpha}=\left[t_{s^{j+1}}^{\alpha}\right]$. Otherwise, since $q_{\alpha} \in \mathcal{Q}$, by definition $x_{i}^{\alpha}$ contains $\left[t_{s^{j}+1}^{\alpha}\right] \subseteq r_{j+1}^{\alpha}$ for all $s^{j}+1<i \leq$ $s^{j+1}$. Hence $\emptyset \neq\left[t_{s^{j}+1}^{\alpha}\right] \cap x_{s^{j}+1}^{\alpha} \subseteq X_{j+1}^{\alpha} \cap r_{j+1}^{\alpha}$ and thus $X_{j+1}^{\alpha}$ splits $r_{j+1}^{\alpha}$.

Therefore the elements $\left\{r_{j}^{\alpha}, X_{j}^{\alpha}: \alpha<\omega_{1}, 0<j<k\right\}$ can be reenumerated as $\left\langle r_{\alpha}, X_{\alpha}: \alpha \in \Omega_{1}\right\rangle$, which satisfies the conditions (1)-(4) of Note 6.10. Thus for our purposes here we can replace $\mathcal{Q}\left\langle\left[t_{\alpha}\right], x_{\alpha}: \alpha \in \Omega\right\rangle$ in Definition 6.9 with its version $\mathcal{Q}\left\langle r_{\alpha}, X_{\alpha}: \alpha \in \Omega_{1}\right\rangle$ since the properties (1)-(4) of Note 6.10 are all the properties of our original sequences needed in this proof. 
Claim 6.14. For each $\alpha<\omega_{1}, q_{\alpha}^{\prime} \in \mathcal{Q}\left\langle r_{\alpha}, X_{\alpha}: \alpha \in \Omega_{1}\right\rangle$. Furthermore, the set $R^{\prime}=\left\{q_{\alpha}^{\prime}: \alpha<\omega_{1}\right\}$ is a strong antichain in $\mathcal{Q}$.

Proof. We first show that $q_{\alpha}^{\prime}$ satisfies the condition of Definition 6.9 for $\left(r_{\alpha}, X_{\alpha}: \alpha \in \Omega_{1}\right)$. Let $\alpha<\omega_{1}$ and $0<j, j^{\prime}<k$. Assume $r_{j}^{\alpha}<_{\text {Lex }} r_{j^{\prime}}^{\alpha}$. Then for any $s^{j}<i \leq s^{j+1}$ and all $s^{j^{\prime}}<i^{\prime} \leq s^{j^{\prime}+1}$ we have $r_{j}^{\alpha} \subseteq\left[t_{i}^{\alpha}\right]$ and $\left[t_{i}^{\alpha}\right] \subseteq x_{i^{\prime}}^{\alpha}$. Thus $r_{j}^{\alpha} \subseteq \bigcap_{s^{j^{\prime}}<i^{\prime} \leq s^{j^{\prime}+1}} x_{i^{\prime}}^{\alpha}=X_{j^{\prime}}^{\alpha}$ as required.

We now show that $R^{\prime}$ is a strong antichain. Let $\alpha \neq \beta<\omega_{1}$. Then, as we have seen above, there exists an $0<i \leq n$ such that $\left[t_{i}^{\alpha}\right]<_{\text {Lex }}\left[t_{i}^{\beta}\right]$ but $\left[t_{i}^{\alpha}\right] \nsubseteq x_{i}^{\beta}$. Let $0<j \leq k$ be such that both $\left[t_{i}^{\alpha}\right]$ and $\left[t_{i}^{\beta}\right]$ extend $r_{j}$. Then $\left[t_{i}^{\alpha}\right]<_{\text {Lex }}\left[t_{i}^{\beta}\right]$ implies that $r_{j}^{\alpha}<_{\text {Lex }} r_{j}^{\beta}$. Moreover, $x_{i}^{\beta} \cap r_{j}^{\alpha}=\emptyset$ implies that $X_{j}^{\beta} \cap r_{j}^{\alpha}=\emptyset$. So $q_{\alpha}^{\prime}$ is incompatible with $q_{\beta}^{\prime}$ as desired. The claim follows.

The previous proof illustrates how $R^{\prime}$ represents $R$. Hence $k$ can be taken to be $n$. From now on we will assume $k=n$ for the strong antichain $R$ of $\mathcal{Q}\left\langle\left[t_{\alpha}\right], x_{\alpha}: \alpha \in \Omega\right\rangle$, which we started with at the beginning of the proof.

Since $\gamma<\omega_{1}$ is such that all the elements $r_{1}, \ldots, r_{n}$ belong to $A_{\gamma}$, we can define a sequence $\left\langle C_{1}, \ldots, C_{n}\right\rangle \in M_{\gamma} \cap \mathcal{B}^{n}$ separating $r_{1}, \ldots, r_{n}$ and, therefore, $R$ as in the proof of Lemma 5.5.

The fact that $R$ consists of pairwise incompatible conditions of $\mathcal{Q}$ means that for $\gamma<\alpha \neq \beta<\omega_{1}$ there exists an $0<i \leq n$ such that $\left[t_{i}^{\alpha}\right]<_{\text {Lex }}\left[t_{i}^{\beta}\right]$ but $\left[t_{i}^{\alpha}\right] \not \subset x_{i}^{\beta} \cap C_{i}$, which in turn implies $x_{i}^{\alpha} \cap C_{i} \not \subset x_{i}^{\beta} \cap C_{i}$.

In other words, if for all $0<i \leq n$ we have $x_{i}^{\alpha} \cap C_{i} \subseteq x_{i}^{\beta} \cap C_{i}$, then there must exist a $0<j \leq n$ such that $\left[t_{j}^{\beta}\right]<_{\text {Lex }}\left[t_{j}^{\alpha}\right]$.

We now define, for $\alpha<\omega_{1}$,

$$
a_{\alpha}=\bigcup_{0<i \leq n}\left(x_{i}^{\alpha} \cap C_{i}\right) .
$$

Then whenever $\gamma<\alpha \neq \beta$ and $a_{\alpha} \subseteq a_{\beta}$ there exists an $0<i \leq n$ such that $\left[t_{i}^{\beta}\right]<_{\text {Lex }}\left[t_{i}^{\alpha}\right]$. Identifying a member $q_{\alpha}$ of $R$ with the $n$-tuple $\left\langle\left[t_{1}^{\alpha}\right], \ldots,\left[t_{n}^{\alpha}\right]\right\rangle$ which enumerates it according to $<_{\text {Lex }}$ we can take $R$ to be a subset of the Cartesian cube $A^{n}$. By our assumption $\left(A,<_{\text {Lex }}\right)$ is a Countryman ordering, so $A^{n}$ is the union of countably many chains. So going to an uncountable subset of $R$ we may in fact assume that $R$ is a chain in $A^{n}$. It follows that for $\gamma<\alpha \neq \beta$, the inclusion relation $a_{\alpha} \subseteq a_{\beta}$ implies that $\left[t_{1}^{\beta}\right]<_{\text {Lex }}\left[t_{1}^{\alpha}\right]$. The lemma follows.

Hence from now on we may assume that (some version of) $\mathcal{Q}$ is c.c.c. Therefore we finish via the following.

Lemma 6.15. $\left(\mathrm{MA}_{\omega_{1}}\right)$ Assume that a version of $\mathcal{Q}$ is c.c.c. Then there exists an uncountable subset of $\mathcal{B}$ which can be extended to a Countryman order. 
Proof. It suffices to show this for the original version of $\mathcal{Q}$ since the properties (1)-(4) of Note 6.10 are all that is needed here. Applying $\mathrm{MA}_{\omega_{1}}$ to $\mathcal{Q}$ gives us an uncountable subset $\Omega_{1}$ of $\Omega$ such that if $\alpha \neq \beta$ are in $\Omega_{1}$ then $\left[t_{\beta}\right]<_{\text {Lex }}\left[t_{\alpha}\right]$ implies $\left[t_{\beta}\right] \subseteq x_{\alpha}$ and, therefore, $x_{\alpha} \nsubseteq x_{\beta}$. This means that $x_{\alpha} \subseteq x_{\beta}$ implies $\left[t_{\alpha}\right]<_{\text {Lex }}\left[t_{\beta}\right]$. The lemma follows.

We now justify the use of a Countryman lexicographical ordering on $A$.

Lemma 6.16. $\left(\mathrm{MA}_{\omega_{1}}\right)$ Let $S$ and $T$ be Aronszajn trees. Then there exists an uncountable subset $\Gamma$ of $\omega_{1}$, a downwards closed uncountable subtree $S_{0} \subseteq S$ and a strictly increasing level-preserving map $f: S_{0}\lceil\Gamma \rightarrow T$.

Note 6.17. In particular, if $T$ has a Countryman lexicographical order then we can define a Countryman lexicographical order on $S_{0}$. Thus if we apply Lemma 6.16 to the downward closure of the set $\left\{\left[t_{\alpha}\right]: \alpha \in \Omega\right\} \subseteq A$, we get an uncountable subset $\Gamma$ of $\Omega$ and a lexicographical ordering $<_{\text {Lex }}$ on the downward closure of $\left\{\left[t_{\alpha}\right]: \alpha \in \Gamma\right\}$ such that $\left(\left\{\left[t_{\alpha}\right]: \alpha \in \Gamma\right\},<_{\text {Lex }}\right)$ is Countryman.

Proof (of Lemma 6.16). Let $\mathcal{R}$ be the poset of finite partial strictly increasing level-preserving functions $p$ from $S$ into $T$ such that $D_{p}=\operatorname{dom}(p)$, and therefore $R_{p}=\operatorname{ran}(p)$, have the following properties:

(a) $D_{p} \subseteq S\left\lceil\Gamma_{p}\right.$ (respectively, $R_{p} \subseteq T\left\lceil\Gamma_{p}\right.$ ) for some finite set $\Gamma_{p} \subseteq \omega_{1}$,

(b) every node of $D_{p}$ has extensions in $D_{p}$ in all levels of $\Gamma_{p}$ above its own (and similarly, this is true also for $R_{p}$ ),

(c) every node of $D_{p}$ has predecessors in $D_{p}$ in all levels of $\Gamma_{p}$ below its own (and similarly, this is true also for $R_{p}$ ).

Assume $\mathcal{E}$ is a given uncountable subset of $\mathcal{R}$. Using the standard $\Delta$ system argument we may assume that $D_{p}(p \in \mathcal{E})$ forms a $\Delta$-system with root $D$, that $R_{p}(p \in \mathcal{E})$ forms a $\Delta$-system with root $R$, and that $\Gamma_{p}(p \in \mathcal{E})$ forms a $\Delta$-system with root $\Gamma^{\prime}$. Moreover we may assume that for $p \neq q$ in $\mathcal{E}$, every ordinal from $\Gamma_{p} \backslash \Gamma^{\prime}$ is smaller than every ordinal from $\Gamma_{q} \backslash \Gamma^{\prime}$, or vice versa. By Lemma 6.5 , we can find $p \neq q$ in $\mathcal{E}$ such that every node of $D_{p} \backslash D$ is incomparable with every node in $D_{q} \backslash D$ and, similarly, every node of $R_{p} \backslash R$ is incomparable with every node in $R_{q} \backslash R$. Supplement $D_{p} \cup D_{q}$ to some subtree $D_{r} \subseteq S \uparrow\left(\Gamma_{p} \cup \Gamma_{q}\right)$ satisfying (a), (b) and (c), and extend $p$ and $q$ to some $r: D_{r} \rightarrow R_{r}$, where $R_{r} \subseteq T \uparrow\left(\Gamma_{p} \cup \Gamma_{q}\right)$ also satisfies (a), (b) and (c), such that $r$ is strictly increasing and level-preserving. Then $r \in \mathcal{R}$ and $r \leq p, q$. This shows that $\mathcal{R}$ is a c.c.c. poset. For $\alpha<\omega_{1}$ let

$$
D_{\alpha}=\left\{p \in \mathcal{R}: \Gamma_{p} \text { contains some ordinal }>\alpha\right\} .
$$

It is easily seen that each $D_{\alpha}$ is a dense open set of $\mathcal{R}$. Let $\mathcal{G} \subseteq \mathcal{R}$ be a $\left\{D_{\alpha}: \alpha<\omega_{1}\right\}$-generic filter. Let $f=\bigcup \mathcal{G}, \Gamma=\bigcup_{p \in \mathcal{G}} \Gamma_{p}$ and $S_{0}$ be the downward closure of $\bigcup_{p \in \mathcal{G}} D_{p}$. The lemma follows. 


\section{References}

[1] U. Abraham and S. Shelah, Martin's axiom does not imply that every two $\aleph_{1}$ dense sets of reals are isomorphic, Israel J. Math. 38 (1981), 161-176.

[2] J. E. Baumgartner, Chains and antichains in $\mathcal{P}(\mathbb{N})$, J. Symbolic Logic 45 (1980), 85-92.

[3] - Applications of the proper forcing axiom, in: Handbook of Set-Theoretic Topology, Elsevier, Amsterdam, 1984, 913-959.

[4] J. E. Baumgartner and P. Komjáth, Boolean algebras in which every chain and antichain is countable, Fund. Math. 111 (1981), 125-133.

[5] R. Bonnet and S. Shelah, Narrow Boolean algebras, Ann. Pure Appl. Logic 28 (1985), 1-12.

[6] B. Dushnik and E. W. Miller, Partially ordered sets, Amer. J. Math. 63 (1941), 600-610.

[7] D. Fremlin, Consequences of Martin's Axiom, Cambridge Univ. Press, 1984.

[8] -, Problems, version of May, 1998.

[9] F. Galvin and B. Jónsson, Distributive sublattices of a free lattice, Canad. J. Math. 13 (1961), 265-272.

[10] Dj. Kurepa, Transformations monotones des ensembles partiellement ordonnés, Rev. Cienc. (Lima) 437 (1943), 483-500.

[11] J. D. Monk, Cardinal Invariants on Boolean Algebras, Birkhäuser, Basel, 1996.

[12] S. Shelah, Decomposing uncountable squares to countably many chains, J. Combin. Theory Ser. A 21 (1976), 110-114.

[13] - On uncountable Boolean algebras with no uncountable pairwise comparable or incomparable sets of elements, Notre Dame J. Formal Logic 22 (1984), 301-308.

[14] W. Sierpiński, Sur un problème de la théorie des relations, Ann. Scuola Norm. Sup. Pisa (2) 2 (1933), 285-287.

[15] S. Todorčević, Trees and linearly ordered sets, in: Handbook of Set-Theoretic Topology, Elsevier, Amsterdam, 1984, 235-293.

[16] - Remarks on chain conditions in products, Compositio Math. 55 (1985), 295-302.

[17] —, Remarks on cellularity in products, ibid. 57 (1986), 357-372.

[18] -, Partitioning pairs of countable ordinals, Acta Math. 159 (1987), 261-294.

[19] -, Irredundant sets in Boolean algebras, Trans. Amer. Math. Soc. 339 (1993), 35-44.

Department od Mathematics

University of Toronto

Toronto, Ontario

Canada M5S 3G3

E-mail: losada@math.toronto.edu stevo@math.toronto.edu
C.N.R.S. (ESA 753)

Université Paris 7

2, Place Jussieu 75251 Paris Cedex 05

France

E-mail: stevo@math.jussieu.fr

Received 5 May 1999;

in revised form 29 October 1999 